\title{
Seismo Electric Field Fractal Dimension for Characterizing Shajara Reservoirs of the Permo-Carboniferous Shajara Formation, Saudi Arabia
}

\begin{abstract}
AlKhidir KEME*
Department of petroleum and Natural Gas Engineering, College of Engineering, King Saud University, Saudi Arabia
\end{abstract}

*Corresponding author: Professor Khalid Elyas Mohamed Elameen AlKhidir, Department of petroleum and Natural Gas Engineering, College of Engineering, King Saud University, Saudi Arabia, Email: kalkhidir@ksu.edu.sa

\section{Research Article}

Volume 2 Issue 4

Received Date: April 30, 2018

Published Date: May 09, 2018

\section{Abstract}

The quality of a reservoir can be described in details by the application of seismo electric field fractal dimension. The objective of this research is to calculate fractal dimension from the relationship among seismo electric field, maximum seismo electric field and wetting phase saturation and to confirm it by the fractal dimension derived from the relationship among capillary pressure and wetting phase saturation. In this research, porosity was measured on real collected sandstone samples and permeability was calculated theoretically from capillary pressure profile measured by mercury intrusion techniques. Two equations for calculating the fractal dimensions have been employed. The first one describes the functional relationship between wetting phase saturation, seismo electric field, maximum seismo electric field and fractal dimension. The second equation implies to the wetting phase saturation as a function of capillary pressure and the fractal dimension. Two procedures for obtaining the fractal dimension have been developed. The first procedure was done by plotting the logarithm of the ratio between seismo electric field and maximum seismo electric field versus logarithm wetting phase saturation. The slope of the first procedure = 3- Df (fractal dimension). The second procedure for obtaining the fractal dimension was completed by plotting the logarithm of capillary pressure versus the logarithm of wetting phase saturation. The slope of the second procedure = Df -3 . On the basis of the obtained results of the constructed stratigraphic column and the acquired values of the fractal dimension, the sandstones of the Shajara reservoirs of the Shajara Formation were divided here into three units. The gained units from bottom to top are: Lower Shajara Seismo Electric Field Fractal Dimension Unit, Middle Shajara Seismo Electric Field Fractal dimension Unit, and Upper Shajara Seismo Electric Field Fractal Dimension Unit. The results show similarity between seismo electric field 


\section{Petroleum \& Petrochemical Engineering Journal}

fractal dimension and capillary pressure fractal dimension. It was also noted that samples with wide range of pore radius were characterized by high values of fractal dimension due to an increase in their connectivity and seismo electric field. In our case, and as conclusions the higher the fractal dimension, the higher the permeability, the better the shajara reservoir characteristics.

Keywords: Shajara reservois; Shajara Formation; Seismo electric field fractal dimension

\section{Introduction}

Seismo electric effects related to electro kinetic potential, dielectric permitivity, pressure gradient, fluid viscosity, and electric conductivty was first reported by Frenkel J [1]. Capillary pressure follows the scaling law at low wetting phase saturation was reported by $\mathrm{Li} \mathrm{K}$, et al. [2]. Seismo electric phenomenon by considering electro kinetic coupling coefficient as a function of effective charge density, permeability, fluid viscosity and electric conductivity was reported by Revil A, et al. [3]. The magnitude of seismo electric current depends porosity, pore size, zeta potential of the pore surfaces, and elastic properties of the matrix was investigated by Dukhin A, et al. [4]. The tangent of the ratio of converted electic field to pressure is approximately in inverse proportion to permeability was studied by Guan $\mathrm{W}$, et al. [5]. Permeability inversion from seismoelectric log at low frequency was studied by $\mathrm{Hu} \mathrm{H}$, et al. [6]. They reported that, the tangent of the ratio among electric excitation intensity and pressure field is a function of porosity, fluid viscosity, frequency, tortuosity, fluid density and Dracy permeability. A decrease of seismo electric frequencies with increasing water content was reportet by Borde $\mathrm{C}$, et al. [7]. An increase of seismo electric transfer function with increasing water saturation was studied by Jardani A, et al. [8]. An increase of dynamic seismo electric transfer function with decreasing fluid conductivity was described by Holzhauer J, et al. [9]. The amplitude of seismo electric signal increases with increasing permeability which means that the seismo electric effects are directly related to the permeability and can be used to study the permeability of the reservoir was illustrated by Rong P, et al. [10]. Seismo electric coupling is frequency dependent and decreases expontialy when frequency increases was demonstrated by Djuraev U, et al. [11]. An increase of permeability with increasing pressure head and bubble pressure fractal dimension was reported by Alkhidir KEME [12]. An increase of geometric and arithmetic relaxtion tiome of induced polarization fractal dimension with permeability increasing was described by Alkhidir KEME [13].

\section{Material and Methods}

Porosity was measured on collected sandstone samples and permeability was calculated from the measured capillary pressure by mercury intrusion techniques. Two procedures for obtaining the fractal dimension have been developed. The first procedure was done by plotting the logarithm of the ratio between seismo electric field and maximum seismo electric field versus logarithm wetting phase saturation. The slope of the first procedure $=3-\mathrm{Df}$ (fractal dimension). The second procedure for obtaining the fractal dimension was completed by plotting the logarithm of capillary pressure versus the logarithm of wetting phase saturation. The slope of the second procedure $=$ Df -3 .

The seismo electric field can be scaled as

$$
S w=\left[\frac{E^{\left[\frac{1}{3}\right]}}{E_{\max }^{\left[\frac{1}{3}\right]}}\right]^{[3-D f]} \quad 1
$$

Where $S_{w}$ the water saturation, $E$ the seismo electric field in volt / meter, $E_{\max }$, the maximum seismo electric field in volt / meter, and Df the fractal dimension.

Equation 1 can be proofed from

$$
\mathbf{E}=\left[\frac{\varepsilon \mathbf{f} * \zeta * \boldsymbol{\rho f} * \ddot{u}}{\boldsymbol{\eta} * \boldsymbol{\sigma f}}\right] \mathbf{2}
$$

Where E the seismo electric field in volt / meter, $\varepsilon f$ dielectric permittivity of the fluid, $\zeta$ the zeta potential in volt, $\rho$ d density of the fluid in kilogram / cubic meter, $\ddot{v}$ the seismo electric acceleration in meter / second square, $\eta$ 


\section{Petroleum \& Petrochemical Engineering Journal}

the fluid viscosity in pascal second, and of the fluid conductivity in Siemens /meter.

$$
\text { But, } \frac{\varepsilon f * \zeta}{\eta * \sigma f}=C_{S} 3
$$

Where $C_{S}$, the streaming potential coefficient in volt / pascal

Insert equation 3 into equation 2

$$
\mathbf{E}=\mathbf{C}_{\mathbf{s}} * \boldsymbol{\rho f} * \ddot{\mathrm{u}} \mathbf{4}
$$

The streaming potential can be scaled as

$$
C_{S}=\frac{V}{Q} \mathbf{5}
$$

Where $\mathrm{V}$ the volume in cubic meter, $\mathrm{Q}$ the electric charge in coulomb Insert equation 5 into Equation 4

$$
\mathbf{E}=\left[\frac{\mathbf{V} * \boldsymbol{\rho f} * \ddot{\mathrm{u}}}{\mathbf{Q}}\right] \mathbf{6}
$$

The volume $\mathrm{V}$ can be scaled as

$$
\mathrm{V}=\frac{4}{3} * 3.14 * \mathrm{r}^{3} 7
$$

Where $r$ the pore radius in meter Insert equation 7 into equation 6

$$
\mathbf{E}=\left[\frac{4 * 3.14 * \mathbf{r}^{3} * \rho \mathbf{f} * \ddot{\mathrm{u}}}{3 * \mathbf{Q}}\right] \mathbf{8}
$$

The maximum pore radius $r_{\max }$ can be scaled as

$$
\mathbf{E}_{\max }=\left[\frac{4 * 3.14 * \mathbf{r}_{\max }^{3} * \rho \mathbf{f} * \ddot{\mathrm{v}}}{3 * \mathbf{Q}}\right] \mathbf{9}
$$

Divide equation 8 by equation 9

$$
\left[\frac{\mathbf{E}}{\mathbf{E}_{\max }}\right]=\left[\frac{\frac{4 * 3.14 * \mathbf{r}^{3} * \rho f * \ddot{u}}{3 * Q}}{\frac{4 * 3.14 * \mathbf{r}_{\text {max }}^{3} * \rho f * \ddot{u}}{3 * Q}}\right] 10
$$

Equation 10 after simplification will become

$$
\left[\frac{E}{E_{\text {max }}}\right]=\left[\frac{r^{3}}{r_{\max }^{3}}\right] 11
$$

Take the third root of equation 11

$$
\sqrt[3]{\left[\frac{E}{E_{\text {max }}}\right]}=\sqrt[3]{\left[\frac{r^{3}}{r_{\text {max }}^{3}}\right]} 12
$$

Equation 12 after simplification will become

$$
\left[\frac{E^{\left[\frac{1}{3}\right]}}{E_{\text {max }}^{\left[\frac{1}{3}\right]}}\right]=\left[\frac{r}{r_{\text {max }}}\right] 13
$$

\section{But, $\log \left[r / r_{\max }\right]=\log S_{w} /[3-D f] 14$}

Insert equation 14 into equation 13

$$
\log S w /[3-D f]=\log \left[E^{1 / 3} / E_{\max }^{1 / 3}\right] 15
$$

Equation 15 after log removal will become

$$
S_{W}=\left[\frac{E^{\left[\frac{1}{3}\right]}}{E_{\text {max }}^{\left[\frac{1}{3}\right]}}\right]^{[3-D f]} 16
$$

Equation 16 the proof of equation 1 which relates the water saturation, seismo electric field, maximum seismo electric field, and the fractal dimension.

The capillary pressure can be scaled as

$$
\log \mathrm{Sw}=(\mathrm{Df}-3) * \log P \mathrm{c}+\text { constant } 17
$$

Equation 17 can be proofed from the number of pores theorey

$$
\mathrm{N}(\mathrm{r}) \propto \mathbf{r}^{-\mathrm{Df}} \mathbf{1 8}
$$

Where $N(r)$ number of pores, $r$ the pore throat radius and $\mathrm{D}_{\mathrm{f}}$ is the fractal dimension.

$$
\text { But, } N(r)=\left[\frac{V_{H g}}{V_{\text {cell }}}\right] 19
$$

Where $\mathrm{V}_{\mathrm{Hg}}$ the volume of mercury intruded the pores, $\mathrm{V}_{\text {cell }}$ the volume of unit cell.

Insert equation 19 into equation 18

$$
\left[\frac{V_{H g}}{V_{\text {cell }}}\right] \propto \mathbf{r}^{-D f} \mathbf{2 0}
$$

If we consider the unit cell as sphere, then the volume of sphere can be scaled as: 


\section{Petroleum \& Petrochemical Engineering Journal}

$$
\mathrm{V}_{\text {cell }}=\frac{4}{3} * 3.14 * \mathbf{r}^{3} 21
$$

Insert equation 21 into equation 20

$$
\left[\frac{\mathbf{V}_{\mathrm{Hg}}}{\frac{4}{3} * 3.14 * \mathbf{r}^{3}}\right] \propto \mathbf{r}^{-\mathrm{Df}} 22
$$

Equation 22 can also be written as

$$
\mathbf{V}_{\mathrm{Hg}} \propto \mathbf{r}^{3-\text { Df }} 23
$$

The pore radius can be scaled as

$$
\mathbf{r}=\frac{2 * \sigma * \cos \theta}{\mathbf{P}_{\mathrm{c}}} 24
$$

Where $\sigma$ mercury surface tension, $\theta$ mercury contact angle and $\mathrm{p}_{\mathrm{c}}$ the capillary pressure.

Insert equation 24 into equation 23

$$
\mathbf{V}_{\mathrm{Hg}} \propto \mathbf{P}_{\mathrm{c}}{ }^{\text {Df-3 }} 25
$$

Differentiate equation 25 with respect to Pc

$$
\frac{\mathrm{dV}_{\mathrm{Hg}}}{\mathrm{dP}_{\mathrm{c}}} \propto(\mathrm{Df}-3) * \mathbf{P}_{\mathrm{c}}^{\mathrm{Df}-4} 26
$$

If we remove the proportionality sign from equation 26 we have to multiply by a constant

$$
\frac{d V_{H g}}{d P_{c}}=\text { constant } * P_{c}^{\text {Df-4 }} 27
$$

If wetting phase saturation (water saturation) is introduced, then equation 27 will be written as

$$
\frac{d S_{w}}{d P_{c}}=\text { constant } * P_{c}{ }^{D f-4} 28
$$

Integrate equation 28

$$
\int \mathrm{dS}_{\mathrm{w}}=\int \operatorname{constant} * \mathbf{P}_{\mathrm{c}}^{\mathrm{Df}-4} * \mathrm{dP}_{\mathrm{c}} 29
$$

Equation 29 after integration will become

$$
S_{w}=\text { constant } * P_{c}{ }^{D f-3} 30
$$

Take the logarithum of equation 30

$$
\log S_{w}=(D f-3) * \log P_{c}+\text { constant } 31
$$

Equation 31 the proof of equation 17 which relates the water saturation, capillary pressure and the fractal dimension.

\section{Results and Discussion}

Based on field observation the Shajara Reservoirs of the Permo-Carboniferous Shajara Formation were divided here into three units as described in Figure 1. These units from bottom to top are: Lower, Middle, and Upper Shajara Reservoir. Their acquired results of the seismo electric fractal dimension and capillary pressure fractal dimension are displayed in Table 1. Based on the attained results it was found that the seismo electric fractal dimension is equal to the capillary pressure fractal dimension. The maximum value of the fractal dimension was found to be 2.7872 assigned to sample SJ13 from the Upper Shajara Reservoir as verified in Table 1. Whereas the minimum value of the fractal dimension 2.4379 was reported from sample SJ3 from the Lower Shajara reservoir as displayed in table 1 . The seismo electric fractal dimension and capillary pressure fractal dimension were observed to increase with increasing permeability as proofed in Table1 owing to the possibility of having interconnected channels. 


\section{Petroleum \& Petrochemical Engineering Journal}

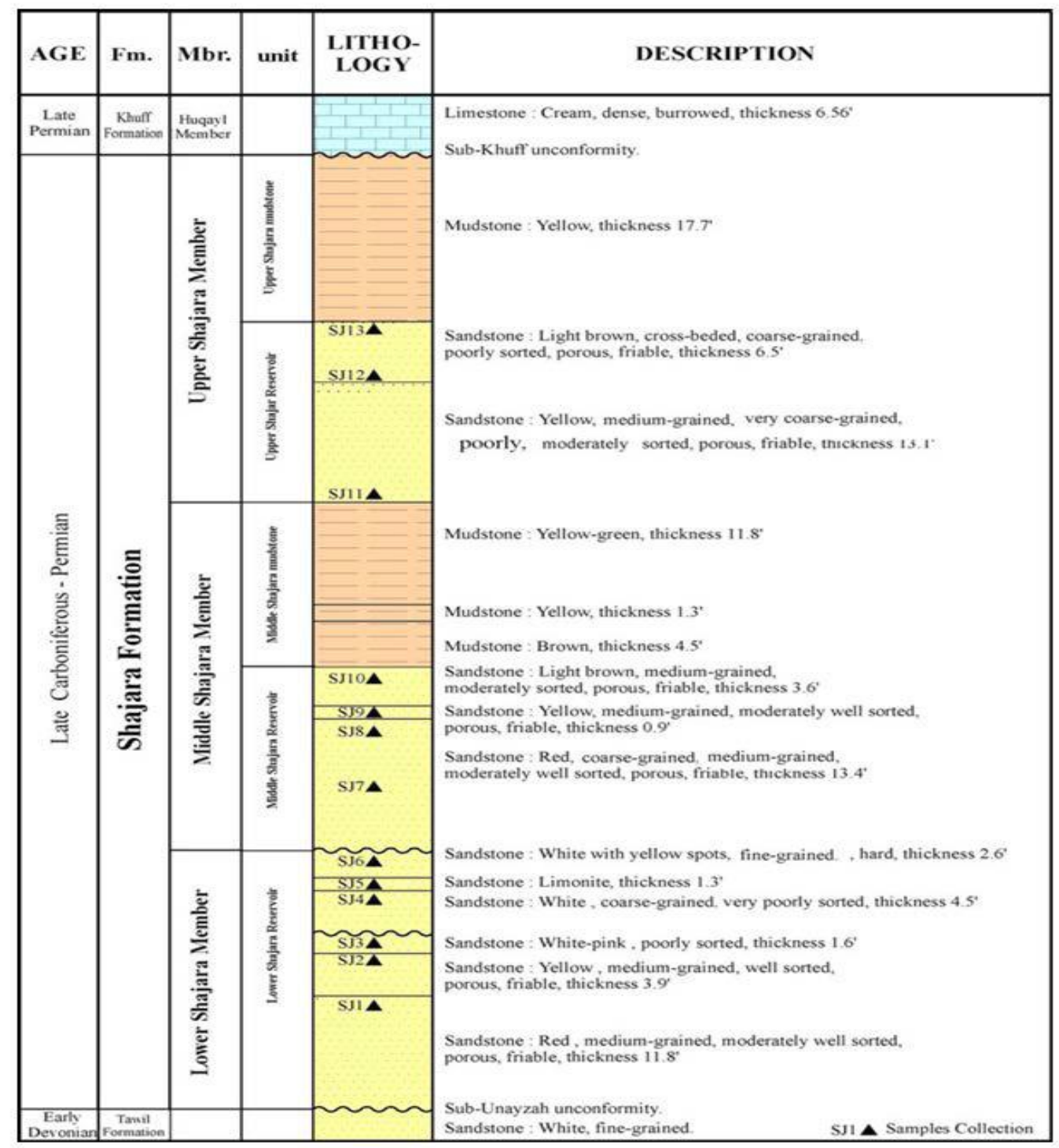

Figure 1: Surface type section of the Shajara Reservoirs of the permo-Carboniferous Shajara Formation, Saudi Arabia latitude $26^{\circ} 52^{\prime} 17.4^{\prime \prime}$ longitude $43^{\circ} 36^{\prime} 18^{\prime \prime}$.

The Lower Shajara reservoir was denoted by six sandstone samples (Figure 1), four of which label as SJ1, SJ2, SJ3 and SJ4 as confirmed in Table1 were selected for capillary measurements. Their positive slopes of the first procedure (log of the ratio of seismo electric field to maximum seismo electric field versus log wetting phase saturation) and negative slopes of the second procedure (log capillary pressure versus log wetting phase saturation are delineated in Table 1. Their seismo electric field fractal dimension and capillary pressure fractal dimension values are shown in Table 1 . As we proceed from sample SJ2 to SJ3 a pronounced reduction in permeability due to compaction was reported from 1955 md to 56 md which reflects decrease in seismo electric field fractal dimension from 2.7748 to 2.4379 as specified in Table 1. Again, an increase in grain size and permeability was verified from sample SJ4 whose seismo electric field fractal dimension and capillary pressure fractal dimension was found to be 2.6843 as described in Table 1. 


\section{Petroleum \& Petrochemical Engineering Journal}

\begin{tabular}{|c|c|c|c|c|c|c|c|}
\hline \multirow[t]{2}{*}{ Reservoir } & \multirow[t]{2}{*}{ sample } & \multirow[t]{2}{*}{$\Phi \%$} & \multirow[t]{2}{*}{ K (md) } & $\begin{array}{c}\begin{array}{c}\text { Postive slope of } \\
\text { the first } \\
\text { procedure }\end{array} \\
\end{array}$ & $\begin{array}{c}\text { negative slope of } \\
\text { the second } \\
\text { procedure }\end{array}$ & \multirow{2}{*}{\begin{tabular}{|} 
Seismo electric \\
field fractal \\
dimension
\end{tabular}} & \multirow{2}{*}{$\begin{array}{c}\text { Pressure } \\
\text { fractal } \\
\text { dimension }\end{array}$} \\
\hline & & & & Slope $=3-\mathrm{Df}$ & Slope $=$ Df -3 & & \\
\hline \multirow{3}{*}{$\begin{array}{l}\text { Upper } \\
\text { Shajara } \\
\text { reservoir }\end{array}$} & SJ13 & 25 & 973 & 0.2128 & -0.2128 & 2.7872 & 2.7872 \\
\hline & SJ12 & 28 & 1440 & 0.2141 & -0.2141 & 2.7859 & 2.7859 \\
\hline & SJ11 & 36 & 1197 & 0.2414 & -0.2414 & 2.7586 & 2.7586 \\
\hline \multirow{3}{*}{$\begin{array}{c}\text { Middle } \\
\text { Shajara } \\
\text { Reservoir }\end{array}$} & SJ9 & 31 & 1394 & 0.2214 & -0.2214 & 2.7786 & 2.7786 \\
\hline & SJ8 & 32 & 1344 & 0.2248 & -0.2248 & 2.7752 & 2.7752 \\
\hline & SJ7 & 35 & 1472 & 0.2317 & -0.2317 & 2.7683 & 2.7683 \\
\hline \multirow{4}{*}{$\begin{array}{c}\text { Lower } \\
\text { Shajara } \\
\text { Reservoir }\end{array}$} & SJ4 & 30 & 176 & 0.3157 & -0.3157 & 2.6843 & 2.6843 \\
\hline & SJ3 & 34 & 56 & 0.5621 & -0.5621 & 2.4379 & 2.4379 \\
\hline & SJ2 & 35 & 1955 & 0.2252 & -0.2252 & 2.7748 & 2.7748 \\
\hline & SJ1 & 29 & 1680 & 0.2141 & -0.2141 & 2.7859 & 2.7859 \\
\hline
\end{tabular}

Table 1: petrophysical model showing the three Shajara reservoirs with their corresponding values of seismo electric fractal dimension and capillary pressure fractal dimension.

In contrast, the Middle Shajara reservoir which is separated from the Lower Shajara reservoir by an unconformity surface as shown in Figure 1. It was designated by four sandstone samples (Figure 1), three of which namely SJ7, SJ8, and SJ9 as illustrated in Table1 were preferred to perform capillary pressure measurements. Their positive slopes of the first procedure and negative slopes of the second procedure are shown in Table 1. Additionally, their seismo electric field fractal dimensions and capillary pressure fractal dimensions show similarities as delineated in Table 1.Their fractal dimension values are higher than those of samples SJ3 and SJ4 from the Lower Shajara Reservoir due to an increase in their permeability as explained in Table 1.

On the other hand, the Upper Shajara reservoir is separated from the Middle Shajara reservoir by yellow green mudstone as revealed in Figure 1. It is defined by three samples so called SJ11, SJ12, SJ13 as explained in Table 1. Their positive slopes of the first procedure and negative slopes of the second procedure are displayed in Table 1. Moreover, their seismo electric field fractal dimension and capillary pressure fractal dimension are also higher than those of sample SJ3 and SJ4 from the Lower Shajara Reservoir due to an increase in their permeability as clarified in Table 1 .

Overall a plot of slopes of the first procedure versus the slopes of the second procedure delineates three permeable zones as presented in Figure 2. These reservoir zones were also proofed by seismo electric field fractal dimension versus capillary pressure fractal dimension as shown in Figure 3. Such variation in fractal dimension can account for heterogeneity which is a key parameter in reservoir quality assessment.

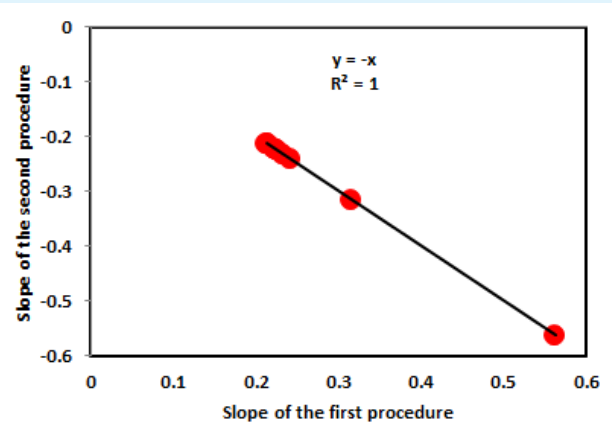

Figure 2: Slope of the first procedure versus the slope of the second procedure.

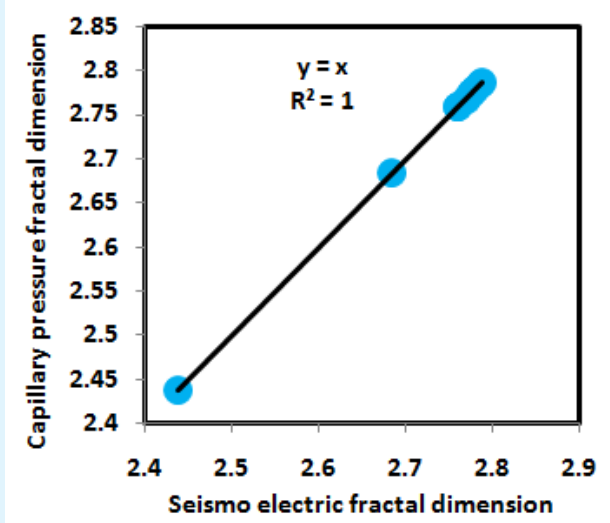

Figure 3: Seismo electric fractal dimension versus capillary pressure fractal dimension. 


\section{Petroleum \& Petrochemical Engineering Journal}

\section{Conclusions}

* The sandstones of the Shajara Reservoirs of the permoCarboniferous Shajara formation were divided here into three units based on seismo electric field fractal dimension.

* The Units from bottom to top are: Lower Shajara seismo electric Field Fractal dimension Unit, Middle Shajara Seismo Electric Field Fractal Dimension Unit, and Upper Shajara Seismoelectric Fractal Dimension Unit.

* These units were also proved by capillary pressure fractal dimension.

* The fractal dimension was found to increase with increasing grain size and permeability.

\section{Acknowledgment}

The author would like to thanks King Saud University, college of Engineering, Department of Petroleum and Natural Gas Engineering, Department of Chemical Engineering, Research Centre at College of Engineering, and King Abdullah Institute for Research and Consulting Studies for their supports.

\section{References}

1. Frenkel J (1944) On the theory of seismic and seismoelectric phenomena in a moist soil. J Physics 3(4): 230-241.

2. Li K, Williams W (2007) Determination of capillary pressure function from resistivity data. Transport in Porous Media 67(1): 1-15.

3. Revil A, Jardani A (2010) Seismoelectric response of heavy oil reservoirs: theory and numerical modelling. Geophysical J International 180(2): 781-797.

4. Dukhin A, Goetz P, Thommes M (2010) Seismoelectric effect: a non-isochoric streaming current. 1 experiment. J Colloid Interface Sci 345(2): 547-553.

5. Guan W, Hu H, Wang Z (2012) Permeability inversion from low-frequency seismoelectric logs in fluid- saturated porous formations. Geophysical Prospecting 61(1): 120-133.

6. Hu H, Guan W, Zhao W (2012) Theoretical studies of permeability inversion from seismoelectric logs. Geophysical Research Abstracts. 14: EGU2012-67251, 2012 EGU General Assembly 2012.

7. Borde C, S'en'echal P, Barri'ere J, Brito D, Normandin $E$, et al. (2015) Impact of water saturation on seismoelectric transfer functions: a laboratory study of co-seismic phenomenon. Geophysical J International 200(3): 1317-1335.

8. Jardani A, Revi A (2015) Seismoelectric couplings in a poroelastic material containing two immiscible fluid phases. Geophysical J International 202(2): 850-870.

9. Holzhauer J, Brito D, Bordes C, Brun Y, Guatarbes B (2016) Experimental quantification of the seismoelectric transfer function and its dependence on conductivity and saturation in loose sand. Geophys Prospect 65(4): 1097-1120.

10. Rong P, Xing W, Rang D, Bo D, Chun L (2016) Experimental research on seismoelectric effects in sandstone. Applied Geophysics 13(3): 425-436.

11. Djuraev U, Jufar S, Vasant P (2017) Numerical Study of frequency-dependent seismoelectric coupling in partially-saturated porous media. MATEC Web of Conferences 87, 02001 (2017).

12. Alkhidir KEME (2017) Pressure head fractal dimension for characterizing Shajara Reservoirs of the Shajara Formation of the Permo-Carboniferous Unayzah Group, Saudi Arabia. Archives Petroleum \& Environ Biotech (2): 1-7.

13. Alkhidir KEME (2018) Geometric relaxation time of induced polarization fractal dimension for characterizing Shajara Reservoirs of the Shajara Formation of the Permo-Carboniferous Unayzah Group, Saudi Arabia. Scifed J Petroleum 2(1): 1-6. 